\title{
PROLONGED COMA AFTER PITUITARY MAGRO- ADENOMA SURGERY: A CASE REPORT WITH SHORT LITERATURE REVIEW
}

\author{
Karki $M^{1}$, Thapa $A J^{1}$, Roka $Y B^{1,2}$
}

${ }^{1}$ Department of Neurosurgery, ${ }^{2}$ Department of Neurosurgery, Neuro-Cardio and Multispecialty Hospital, Skull Base and Neurovascular Research Center, Neuro-Cardio and Multispecialty Hospital, Biratnagar, Morang, Nepal

\begin{abstract}
Prolonged coma after surgery of pituitary macro-adenoma with apoplexy, often previously unnoticed, is rare and not well reported complication. A 65 year male presented with complaints of headache and blurred vision since few months. Brain MRI was done and it reported macro- adenoma with pituitary apoplexy. Right pterional craniotomy was done following total excision of tumor. Patient developed whole body seizure at first day of surgery and went to prolonged coma.
\end{abstract}

\section{KEYWORDS}

Pituitary Macro-adenoma, Surgery, Thalamic Infarction, Vasospasm and Coma

\section{CORRESPONDING AUTHOR}

Dr. Yam Bahadur Roka,

Department of Neurosurgery, Neuro-Cardio and

Multispecialty Hospital, Skull Base and Neurovascular

Research Center, Biratnagar, Morang, Nepal,

Postal code- 56600,

Email: dryamroka@yahoo.com

ORCID ID: 0000-0003-1458-3705 


\section{INTRODUCTION}

Pituitary adenomas constitute approximately $10-15 \%$ of all intracranial tumors, ${ }^{1}$ and can be associated with apoplexy which is a life threating clinical condition with incidence of $0.6-22 \% .^{2}$ Prolonged coma is a very rare clinical presentation after pituitary adenoma surgery the mechanism of which are not fully understood. Some of the factors leading to prolonged coma are due to preoperative adrenal insufficiency, manipulation of the pituitary gland/stalk during surgery, or electrolyte metabolism disorders in pre or post-operative period which can lead to coma for several days or months. Reduction of cyclic adenosine monophosphate (cAMP) in cerebrospinal fluid (CSF), and subnormal level of hypothyroidism and hypogonadism following head trauma are also associated with vegetative state or coma. ${ }^{3}$ Prolonged coma is rarely reported following traumatic or non-traumatic injury to thalamus or/ hypothalamus. ${ }^{4}$ This case report records an uncommon complication of pituitary macro adenoma surgery.

\section{MATERIALS AND METHODS}

A 65 year old male presented in our emergency department with history of headache, dizziness, blurred vision in both eyes, followed by progressive generalized weakness for 5 days.

On examination he was conscious but irritable with intact cranial and systemic neurological system. Fundoscopy did not reveal papilledema. CT scan showed dis-homogeneously hyper -dense intrasellar lesion with uniform contrast uptake. Patient was further investigated with magnetic resonance imaging (MRI) which showed $2.4 \mathrm{x}$ $2.3 \times 2 . c m$ sella mass showing hypointese T1, intermediate to hyperintense T2/FLAIR signal subtle to absent enhancement causing widening of sellar floor with evidence of suprasellar extension abutting the optic chiasma and extending to bilateral cavernous internal carotid arteries (ICA) (Fig. 1). Pituitary hormone profile including, prolactin, growth hormone, thyroid function and cortisol were all within normal values.

In view of the large suprasellar extension and patient preference for transcranial approach, a right pterional craniotomy was performed to remove the tumor. Intraoperatively the Adenoma was large encapsulated with splaying of right optic nerve and extending laterally beyond both ICA with hemorrhagic contents. Microscopic total excision of tumor was done with decompression of both optic nerve and chiasma. Posoperatively the patient was extubated and kept in the ICU

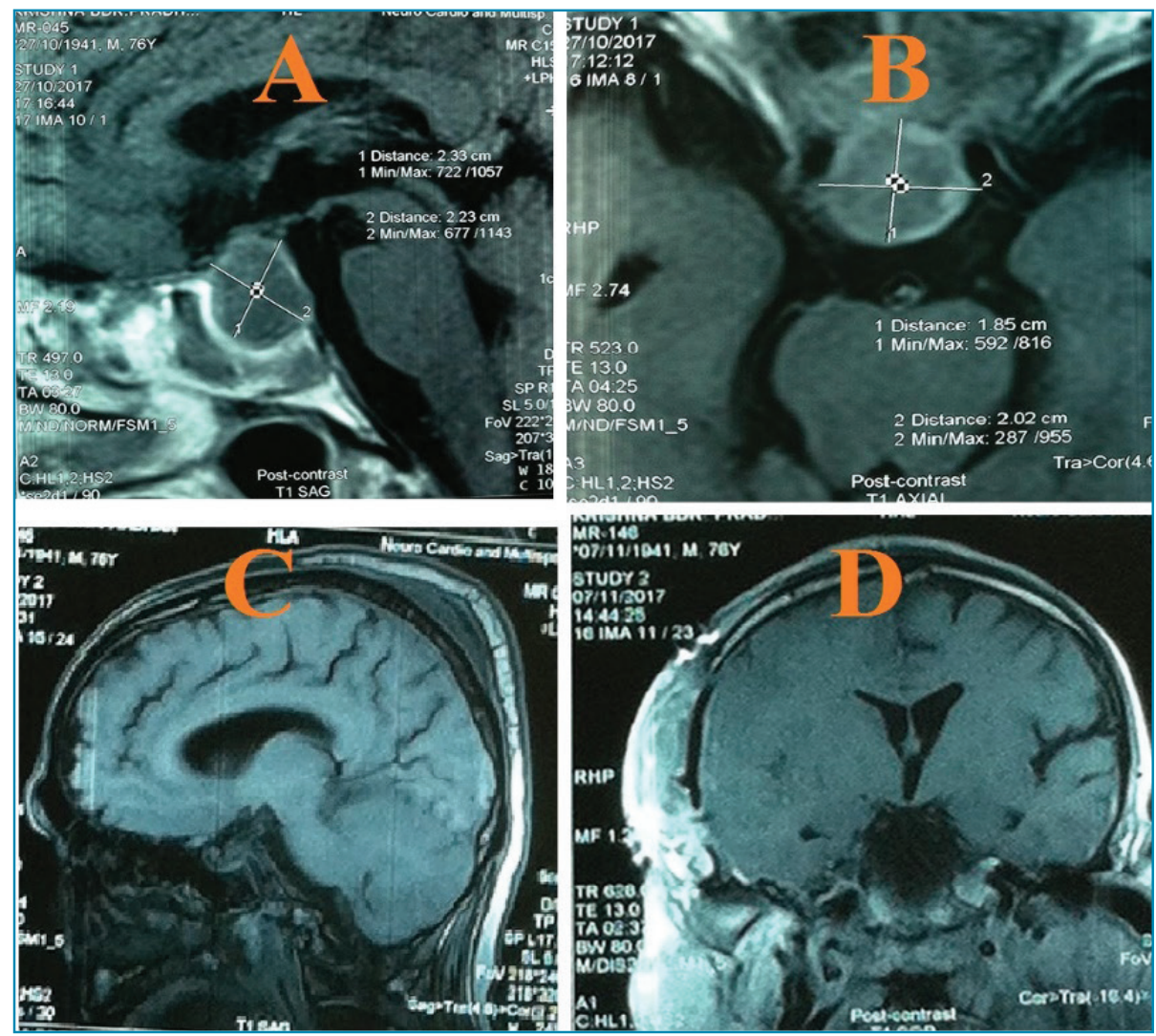

Fig. 1. (A and B) shows Pre-operative Contrast enhanced T1 Sagittal and T1 Axial MRI images respectively with $2.4 \times 2.3 \times 2 . c m$ sellar mass; (C and $\mathbf{D})$ shows post-operative T1 Sagittal and T1 Coronal MRI images respectively. 
where he started to have generalized seizure on second day. CT scan of the head did not reveal any abnormality.

In spite of adequate dosing of antiepileptic medication, the patient developed refractory seizure (status epilepticus) on third day of surgery for which Diazepam in infusion pump was started. Bedside Electro Encephalogram showed generalized spikes suggestive of seizure. He was weaned off Diazepam after a week and continued on levetiracetam with good control of the seizure. The patient remained in persistent comatose state (GCS of 3/5) even after a week of stopping diazepam. His thyroid function test, daily electrolytes and sugar, cardiovascular, renal and hepatic functions were normal. Pupils were reactive to light in both eyes. An MRI brain with Diffusion Weighted images (DW) was done which showed bilateral thin hemorrhage in the lateral ventricles, punctuate bleed in the right cerebral peduncle with partial loss of grey white differentiation in right fronto-temporal and right antero-medial region of thalamus (suggestive of subacute infarction) (Fig. 2). He was started on Clopidogrel (75mg OD) and continued on ventilator support. Three weeks after surgery he started to move the limbs and within five days was conscious and hence removed off ventilator. On $28^{\text {th }}$ day post-surgery he was discharged with GCS of 15 and walking with support without any motor or sensory deficit. Histopathology report

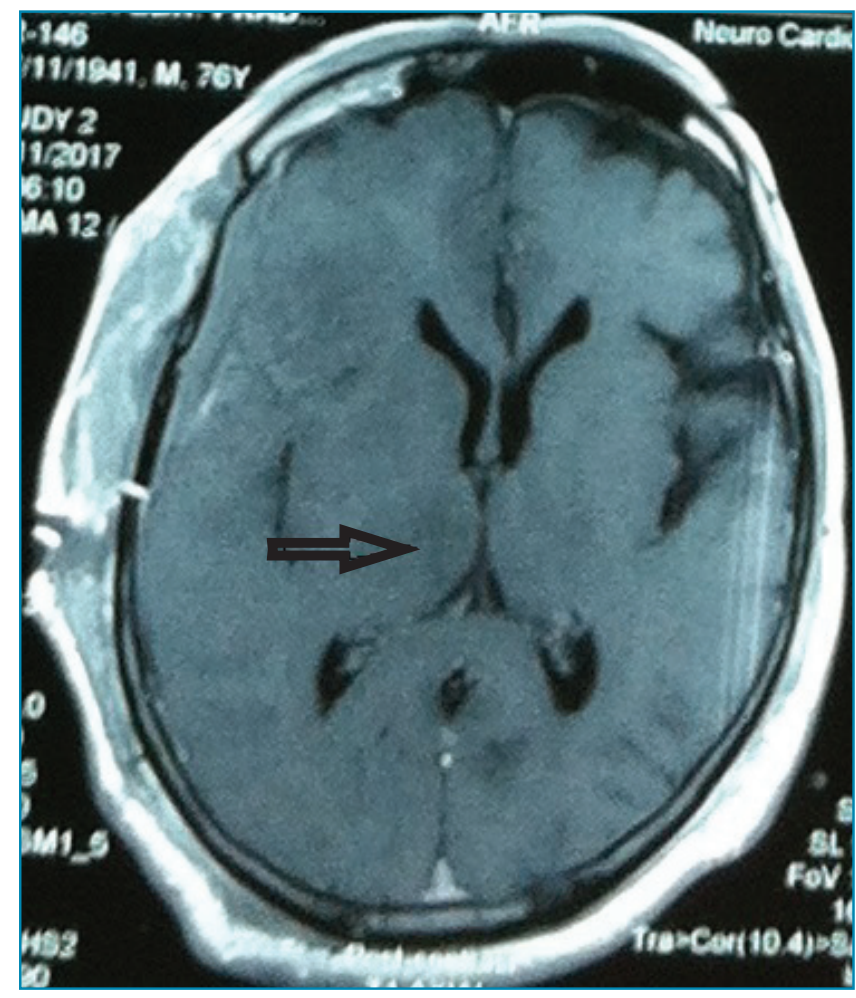

Fig. 2: Shows post-operative T1 Axial MRI image with right fronto -temporal and right thalamus infarct (Arrow). showed pituitary adenoma with apoplexy (Fig. 3). On follow-up at two weeks and 1 month he remains neurologically intact and is on regular follow-up since then.

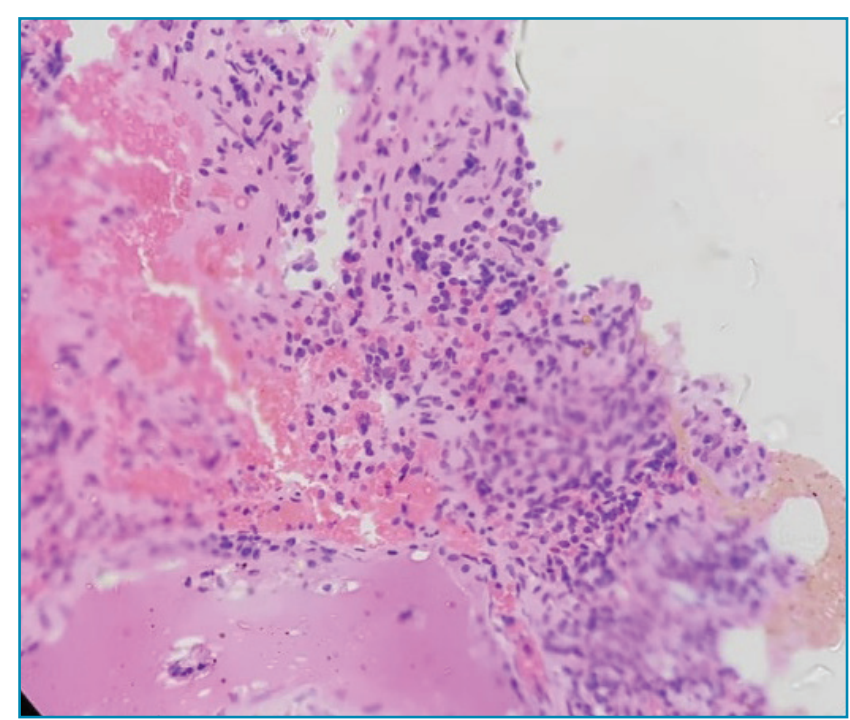

Fig. 3: (H \& E, x 400hpf) section reveals area of hemorrhagic infarct with neoplastic monomorphic population of cells. The cells are round to oval with large nucleus with coarse chromatin pattern and some shows prominent nucleoli and moderate to abundant granular eosinophilic cytoplasm (acidophils).

\section{DISCUSSION}

Pituitary adenomas are one of the most common tumor of the central nervous system with reported annual incidence of $2.63 / 100,000$ person-years and prevalence between 25/100, 00 to 77.6 / 100,000 population. ${ }^{5}$ Pituitary adenomas are further classified either by size into microadenoma $(<10 \mathrm{~mm})$ and macroadenoma $(>10 \mathrm{~mm})$ OR according to hormonal activity as functional and non-functional tumors. These tumors have the potential to compress surrounding structures such as optic nerve and chiasma, carotid arteries, pituitary stalk or hypothalamus, third ventricle and even cranial nerves. Depending on their size, location or extension these tumors may present with visual disturbances, headache or disturbed conscious level, cranial nerve palsies, behavioral changes, seizure, coma, shock and endocrine related dysfunction. ${ }^{6}$ Pituitary apoplexy is the mechanism where acute bleeding occurs within a pituitary adenoma because of excessive tumor infarction, asymptomatically in approximately $10-22 \%$ of patients and symptomatically in 0.69\% with pituitary adenoma needing emergency management. ${ }^{7} \mathrm{CT}$ scan or MRI is the investigation of choice which will reveal the size, site, extension, vascularity, contents and relation of the tumor to the surrounding structures. Fundoscopy and HVF 
helps in defining the visual changes including field defects. Functional tumors especially Prolactinomas can be managed medically but other types require surgery either by transnasal or via transcranial route.

Prolonged coma is very unusual after pituitary surgery. PubMed search for "coma after pituitary adenoma surgery" shows only 18 results with only one study of prolonged coma reported. The latter study by Decker et al. in 1991 reported two cases with progressive coma after transphenoidal surgery.$^{8}$ They postulated that the cause for the coma could be displacement of the $3^{\text {rd }}$ ventricle by residual tumor with increase in hydrocephalus, increase cerebral edema by arginine vasopressin release during manipulation and edema in residual tumor leading to hydrocephalus. They suggest early shunting and radiotherapy to manage these causes.

In our case the patient had intractable seizure for which Diazepam was started in infusion. The sedative effects of diazepam on the central nervous system are well known and initially this was the reason expected. The post-operative repeat CT scan did not show any abnormalities and neither were there any metabolic disturbances which led us to believe that Diazepam was the cause of coma. Only after DW-MRI that showed the thalamic infarct was the cause of coma confirmed. Thalamic infract are notoriously missed because they often cause coma without any focal neurological deficits. They have been reported more often with bilateral thalamic infarcts or hemorrhage and classically present as hypersomnia or sleep-like coma. ${ }^{9}$

The cause of thalamic infarct in this case is probably secondary to perforator vasospasm. Vasospasm in the absence of sub arachnoid hemorrhage is an unusual complication in pituitary surgery. ${ }^{10}$ It has been reported secondary to a variety of surgical approaches such as transsphenoidal, transcranial, fronto-temporal and frontal approaches for various intracranial diseases. ${ }^{11}$ Vasospasm may also lead to cerebral ischemia and/or infarction following pituitary surgery. Mechanical manipulation of the vessels, irritation by surgical instruments, release of some vasoactive mediators from the hypothalamus, and blood loss during surgery may be some of the provoking factors for causes of the vasospasm. ${ }^{12}$ New onset motor deficits (62\%) and changes in mental status (56\%) are most common presenting symptoms in these cases without thalamic infarction. ${ }^{13}$

Intracranial hematoma need to evacuated if developed following pituitary surgery which might produce vasospasm. It is important that adequate preoperative planning need to be conducted with view to understand the relevant anatomy of the local vasculature and minimize this mechanical irritation of vasculatures and hypothalamus which may limit the progression of clinically serious vasospasm In cases with prolonged coma after surgery an early MRI-DW is a must to rule out thalamic or midbrain infarction. ${ }^{14}$ Options for management of such cases involve strict blood pressure maintenance, intravenous tissue plasminogen activator, hematoma evacuation, use of platelet aggregation inhibitors or aspirin and other neuroprotective medications.

Prolonged coma following pituitary surgery is an uncommon and not well reported neurological sequel. We conclude that our patient developed prolonged coma may be due to vasospasm secondary to vessel irritation leading to thalamic infarction. In all cases with prolonged coma with or without focal deficit an MRI must be done to rule out thalamic or midbrain infarction. Timely diagnosis can lead to early intervention and better outcome in such cases.

\section{ACKNOWLEDGEMENTS}

We would like to thank Dr. Sagar Raj Pandey for providing histopathology informations.

\section{REFERENCES}

1. Gittleman H, Ostrom QT, Farah PD et al. Descriptive epidemiology of pituyitary 235 tumors in the united states, 2004-2009: clinical article. J Neurosurg 2014; 121: 527-35 .

2. Kim JP, Park BJ, Kim SB et al. Pituitary apoplexy due to pituitary adenoma infarction. J Korean Neurosurg 2008; 43: 246-49.

3. Fleischer AS, Rudman DR, Payne NS, Tindall GT: Hypothalamic hypothyroidism and hypogonadism in prolonged traumatic coma. J Neurosurg 1978; 49: 650-7.
4. Adams JH, Graham DI, Jennett B. The neuropathology of vegetative state after an acute brain insult. Brain 2000; 123: 13270- 1338.

5. Farnandez A, Karavitaki N, Wass JAH: Prevalence of pituitary adenoma: A community-based, cross sectional study in Banbury (Oxfordshire, UK). Clin Endocrinol (Oxf) 2010; 72: 377-82.

6. Musluman AM, Cansever T, Yilmaz A et al. Surgical results of large and giant pituitary adenoma with special consideration of ophthalmogical outcomes. 
World Neurosurg 2011; 76: 141-8.

7. Bi WL, Dunn IF, Laws LR. Pituitary apoplexy. Endocrine 2015; 48: 69- 75.

8. Decker RE, Chalif DJ: Progressive coma after the transsphenoidal decompression of a pituitary adenoma with marked suprasellar extension: report of two cases. Neurosurgery 1991; 28: 154-7.

9. Honig A, Eliahou R, Eichel R, Shemesh AA, Ben-Hur T, Auriel E. Acute bithalamic infarct manifesting as sleep-like coma: A diagnostic challenge. J Clin Neurosci 2016; 34: 81-5.

10. Page PS, Kim DD, Hall GC, Koutourousiou M. Cerebral vasospasm following endoscopic endonasal surgery for pituitary adenoma resection in the absence of post-operative subarachnoid hemorrhage. J Neurol Stroke 2016; 4: 00130.
11. Papugaev KA, Sabin IA, Lubnin AU et al. Unusual cause of cerebral vasospasm after pituitary surgery. Neurol Sci 2011; 32: 673-80.

12. Kasliwal MK, Srivastava R, Sinha S, Kale SS, Sharma BS. Vasospasm after transsphenoidal pituitary surgery: a case report and review of the literature. Neurol India 2008; 56:81.

13. Mansouri A, Fallah A, Cusimano MD, Das S: Vasospasm post pituitary surgery: systematic review and 3 case presenations. Can J Neurol Sci 2012; 39: 767-73.

14. Honig A, Eliahou R, Eichel R, Shemesh AA, Ben-Hur T, Auriel E. Acute bithalamic infarct manifesting as sleep-like coma: A diagnostic challenge. J Clin Neurosci 2016; 34: 81-5. 